\title{
Adjuvant Radiotherapy
}

National Cancer Institute

\section{Source}

National Cancer Institute. Adjuvant Radiotherapy. NCI Thesaurus. Code C101642.

Radiation therapy that is administered to augment or stimulate other treatment

modalities or to minimize or prevent disease recurrence. 